auf staatliche Mittel zurückgreifen (wobei letzteres von den im Band analysierten nur bei den skandinavischen Parteien im Vordergrund steht).

Den Herausgebern ist es gelungen, mit dieser Zusammenstellung vielfältige Gesichtspunkte des Parteienwandels zu beleuchten. Ihre Einleitung und ihr Schlusskapitel stellen die einzelnen Beiträge in einen Gesamtkontext. Der Vorzug der Vielschichtigkeit hat allerdings einen Nachteil: Die Heterogenität lässt keine einheitliche Sichtweise zu. Einzelne Beiträge, wie etwa der zur Verteilungspolitik in Berlin (Louise K. Davidson-Smith) passen allenfalls sehr eingeschränkt in das Analyseraster und den Gesamtrahmen dieses ansonsten beachtenswerten Sammelbandes. Für Parteienforscher, aber auch für den an Wandel von Parteien allgemeiner interessierten Leser bietet die Publikation eine Reihe von lesenswerten Informationen und gut begründeten Schlussfolgerungen.

Uwe Jun

\title{
Internationale Wahlbeobachtung als Demokratisierungshilfe
}

Münzing, Ekkehard: Internationale Wahlbeobachtung nach dem Ende des Ost-West-Konflikts. Unter besonderer Berücksichtigung der Afrikanischen Union, des Carter Centers, des Commonwealth of Nations, der Organisation Amerikanischer Staaten und der Organisation für Sicherheit und Zusammenarbeit in Europa, Poli-c-books, Berlin 2005, 328 Seiten, € 34,80.

Gut 15 Jahre nach Ende des Ost-West-Konflikts und dem damit einhergehenden Demokratisierungsschub ist die Legitimität von Wahlen beziehungsweise Wahlprozessen immer noch zentrales Thema der Politik. Allein für das Jahr 2006 sei hier auf die Präsidentschaftswahl in Weißrussland und die Wahl im Kongo - und deren Absicherung durch einen EUMilitäreinsatz unter Beteiligung deutscher Soldaten - hingewiesen. In solchen Situationen rücken die Berichte der Wahlbeobachter in den Fokus der internationalen Öffentlichkeit. Sie gelten als ein Instrument der Demokratisierungshilfe und sind inzwischen so häufig eingesetzt worden, dass mitunter von einer „booming industry“ und „monitoring industry“ (S. 4) gesprochen worden ist.

Die Wahlbeobachtung und die internationalen Institutionen, die sie durchführen, werden in der hier anzuzeigenden lesenswerten Dissertation mit hohem aktuellen politischen Bezug von Ekkehard Münzing einer vergleichenden Analyse und Bewertung unterzogen. Im ersten Teil werden Grundlagen und Hintergründe der Wahlbeobachtung analysiert und eine gründliche theoretische Rechtfertigung für das große Engagement der zahlreichen Akteure geliefert. Aufbauend auf der Kantschen These des demokratischen Friedens wird überzeugend begründet, dass die „Etablierung und Konsolidierung demokratischer Systeme in bisher undemokratischen Staaten im originären Eigeninteresse der demokratischen Staaten “ (S. 18) liegt. Schließlich wird das große Spektrum von Akteuren und Instrumenten der Demokratisierungshilfe herausgearbeitet, bei denen internationale Wahlbeobachtungen nur ein Instrument unter vielen sind.

Münzings Analyse der internationalen und regionalen Menschenrechtsverträge ergibt ein disparates Ergebnis. So hat zwar „eine breite völkerrechtliche Verankerung des Rechts auf 
politischer Teilhabe durch demokratische Wahlen stattgefunden" (S. 94) und die Geltung eines gewohnheitsrechtlichen Mindeststandards an Menschenrechten ist mittlerweile so gut wie unbestritten, allerdings gehöre zu diesem „Grundbestand nicht das Menschenrecht auf demokratische Teilhabe an der Staatsgewalt“ (S. 95).

Einen praktischen Bezug, und deswegen durchaus als Lektüre für Wahlbeobachter zu empfehlen, bietet das Kapitel, in dem die Methoden und Tricks der Wahlfälscher in den einzelnen Phasen des Wahlprozesses anschaulich beschrieben und mit konkreten Beispielen illustriert werden. Geprüft wird, in welcher Phase eines Wahlprozesses die theoretischen Möglichkeiten und praktischen Versuche einer undemokratischen Beeinflussung am stärksten ausgeprägt sind. Mit der Wahlbeobachtung werden von den durchführenden Institutionen mitunter sehr unterschiedliche Ziele beziehungsweise Funktionen verknüpft. Münzing arbeitet zwölf direkt mit dem Wahlprozess verbundene heraus. In einem daran anschließenden kurzen Exkurs werden die Vor- und Nachteile einer Wahlbeobachtung durch einheimische statt durch internationale Beobachter dargestellt.

Die internationale Wahlbeobachtung ist zwar seit einigen Jahren ein viel beachtetes Instrument, dennoch ist kaum bekannt, dass sie auf eine fast 150-jährige Geschichte zurückblicken kann. Die älteste Wahlbeobachtung fand in den Fürstentümern Moldau und Walachei im Jahre 1857 statt, bei der die sieben hinter der Neuordnung der Region stehenden Großmächte die Wahlen und deren Überwachung „nicht aus demokratietheoretischen Überlegungen beziehungsweise Überzeugungen anordneten, sondern schlicht deswegen, weil sie sich nicht auf eine gemeinsame Vorgehensweise einigen konnten" (S. 127).

Im zweiten Teil der Studie wird das Engagement von fünf internationalen Wahlbeobachtungsorganisationen vorgestellt. Es sind dies Organisationen dreier regionaler Abmachungen nach Kapitel VIII der UNO-Charta, nämlich die Afrikanische Union, die Organisation Amerikanischer Staaten und die Organisation für Sicherheit und Zusammenarbeit in Europa sowie ferner das Carter Center und das Commonwealth of Nations. Münzing entwickelt ein - repräsentatives - Muster einer durchgeführten Wahlbeobachtung und stellt Überlegungen zur Reform der jeweiligen Wahlbeobachtungsmethodik vor. In seinem Vergleich der Organisationen wird die „qualitative Überlegenheit“ (S. 269) der OSZE deutlich - sowohl in normativer als auch in materieller Hinsicht.

Die hier vorgestellte Dissertation, die man ohne Übertreibung als konkurrenzloses Standardwerk zur internationalen Wahlbeobachtung bezeichnen kann, überzeugt in zweierlei Hinsicht: Sie ist sowohl für den theoretisch interessierten Wissenschaftler als auch für den Praktiker empfehlenswert. 